\title{
ANÁLISE DA REOLOGIA DE ARGAMASSAS AUTOADENSÁVEIS COM ADIÇÕES MINERAIS DE SÍLICA ATIVA E CINZA DO BAGAÇO DE CANA-DE-AÇÚCAR.
}

Guilherme Machado Balbino; Etienne Tainá Damaceno Ferreira; Vinícius Carrijo Dos Santos; Romel Dias Vanderlei.

Universidade Estadual de Maringá, Departamento de Engenharia Civil, Maringá, PR. E-mail: guilhermebalbino1@live.com.

\section{RESUMO}

O presente trabalho analisa o comportamento de argamassas autoadensáveis no estado fresco para produção de concreto autoadensável ao realizar adições minerais de cinza de bagaço de cana-de-açúcar e sílica ativa. A metodologia utilizada, desenvolvida por Okamura e Ouchi (2003), consiste na análise da deformabilidade da argamassa a partir do teste de mini slump e da viscosidade pelo ensaio funil $\mathrm{V}$ para verificar sua capacidade autoadensável. Pelo ensaio de compacidade determinou-se uma porcentagem ótima de substituição de areia por CBC de 40\%, em massa. A relação aglomerantes/agregados 1:2 conferiu argamassas autoadensáveis sem a ocorrência de exsudação e segregação dos materiais. Então, realizou-se adições de sílica ativa nos teores de $5 \%, 7,5 \%$ e $10 \%$ e concluiu-se que esta adição não influencia significativamente nas propriedades autoadensáveis da argamassa, causando aumento da viscosidade e redução da fluidez.

Palavras-chave: reologia, sílica ativa, cinza do bagaço de cana-de-açúcar, autoadensável, resíduos agroindustriais.

\section{ANALYSE OF RHEOLOGY OF SELF-COMPACTING MORTARS WITH MINERALS ADDITIONS OF ACTIVE SILICA AND SUGARCANE BAGASSE ASH.}

\begin{abstract}
This paper analyses the comportament of self-compacting mortars when is added mineral additions of Sugarcane Bagasse Ash and Active Silica. The metodology used, developed by Okamura and Ouchi (2003), consisting of the analyse of deformability of mortar from the mini slump test and of the analyse of viscosity by funnel $V$ test to verify its self-compacting capacitity. The optimum percentage of sand replace by Sugarcane Bagasse Ash was determinate by compactness test, and it was $40 \%$, by mass. The relation binders/aggregates $1: 2$ produced selfcompacting mortars without exudation and segregation of materials. Then, Active Silica was added in contents $5 \%, 7,5 \%$ and $10 \%$ and it was concluded that this addition does not influence significantly to self-compacting properties of mortar, because it increases viscosity and it reduces the fluidity.
\end{abstract}

Keywords: Rheology, Active Silica, Sugarcane Bagasse Ash, Self-compacting, Agroindustrial leavings. 


\section{INTRODUÇÃO}

A crescente influência da indústria da construção civil na economia ao redor do mundo nos últimos anos gera o aumento na competitividade no setor, bem como o maior consumo de materiais durante os processos. Acompanhando essa tendência, Lima (2014) afirma que o concreto se tornou o material mais utilizados na construção civil. No entanto, a exigência de maior qualidade em estruturas de concreto armado, fez com que surgisse um concreto especial capaz de se adensar sem nenhuma intervenção durante as fases de lançamento e de adensamento, de se apresentar fluido, sem que ocorra segregação dos materiais e exsudação, dando origem a um concreto denominado autoadensável (OKAMURA; OUCHI, 2003).

A correta destinação, bem como o aproveitamento de resíduos industriais é outro assunto amplamente discutido e buscado em diversos setores da economia. Diante disso, e pelo fato de que o Brasil é o maior produtor de cana-de-açúcar do mundo, em consequência dessa alta produção de açúcar, etanol e energia, é gerado o bagaço, o qual é aproveitado em grande parte das usinas como combustível para suas caldeiras, e como resíduo resulta a cinza do bagaço de cana-de-açúcar $(C B C)$, cujo descarte nem sempre é apropriado, sendo muitas vezes feito na natureza. Fato que leva ao interesse na elaboração de trabalhos em busca da aplicação desses resíduos agroindustriais na construção civil, especificamente na substituição de parte da areia, de origem quartzosa, na produção de concretos.

A presença de adições minerais, bem como a utilização de aditivos químicos na composição do concreto autoadensável, são essenciais para que o mesmo apresente alta resistência a segregação, deformabilidade adequada e não ocorra exsudação. Desta forma, o presente estudo tem por objetivo analisar o comportamento das propriedades no estado fresco de argamassas autoadensáveis substituindo parcialmente a areia por cinza do bagaço de cana-de-açúcar e adicionar sílica ativa em sua composição.

\section{MATERIAIS E MÉTODOS}

Para atingir os objetivos deste trabalho foram produzidas argamassas com características autoadensáveis, com a substituição parcial do agregado miúdo por CBC e adição mineral de sílica ativa e com utilização de aditivo superplastificante, a fim de avaliar o comportamento de suas propriedades reológicas no estado fresco devido à variação das relações de sílica ativa/cimento e superplastificante/cimento por meio de ensaios propostos por Okamura e Ouchi (2003).

\section{Materiais}

Foram utilizados para a produção das argamassas os seguintes materiais: agregado miúdo, cimento, água, cinza do bagaço de cana-de-açúcar, aditivo superplastificante e sílica ativa.

O cimento utilizado foi o Cimento Portland de Alta Resistência Inicial (CP V ARI) com massa específica de $3,12 \mathrm{~g} / \mathrm{cm}^{3}$.O agregado miúdo utilizado foi a areia quartzosa. A cinza do bagaço de cana-de-açúcar empregada como substituição parcial a areia na composição do agregado miúdo é proveniente da Usina Santa Terezinha da unidade do distrito de Iguatemi no estado do Paraná. Foi utilizado o aditivo superplastificante de terceira geração, sendo composto por éter policarboxílico que atua como dispersante do material cimentício. Como adição mineral foi utilizada sílica ativa que apresenta massa específica de $2,20 \mathrm{~g} / \mathrm{cm}^{3}$.

\section{Ensaio de composição entre agregado miúdo e cinza do bagaço de cana-de-açúcar}

O procedimento de ensaio consiste na mistura de areia média com cinza do bagaço de cana-de-açúcar (CBC) de 10 em 10\% em massa, até a quantidade dos dois materiais se igualarem. Para a realização do estudo foi utilizado a norma ABNT NBR NM 45: 2006, onde, era executado o ensaio para cada uma das composições analisadas e determinado a massa unitária no estado 
compactado seco, desta maneira, a composição com a maior massa unitária foi eleita para ser utilizada na produção das argamassas.

\section{Dosagens}

A partir da determinação da composição entre agregado miúdo e CBC foram realizados ensaios de argamassas com as relações 1:1, 1:2 e 1:3 (aglomerante/agregado miúdo em massa) e para cada família com essas relações foram analisadas as características autoadensáveis variandose a quantidade de aditivo superplastificante nas proporções de $0 \%, 0,2 \%, 0,4 \%, 0,6 \%, 0,8 \%$ e $1 \%$ em relação a massa de cimento. Por fim, foi definido a melhor relação aglomerante/agregado miúdo por meio dos resultados obtidos nos ensaios de mini cone de espalhamento e mini funil-V para argamassas. Definida a melhor relação de aglomerante/agregado foram realizados ensaios com adição de sílica ativa na proporção de $0 \%, 5 \%, 7,5 \%$ e $10 \%$, onde para cada família foram analisados traços com $0 \%, 0,2 \%, 0,4 \%, 0,6 \%, 0,8 \%$ e $1 \%$ de aditivo superplastificante em relação a massa de cimento.

\section{Métodos de ensaio}

O procedimento para a análise das argamassas constituiu na confecção dos traços dosados e posterior realização de ensaios previstos na metodologia de Okamura e Ouchi (2003). As realizações destes ensaios buscam estabelecer uma argamassa que apresente índices $\mathrm{Gm}$ e $\mathrm{Rm}$ capazes de demonstrar autoadensabilidade, conforme, os limites propostos por Nepomuceno, Oliveira e Lopes (2012) e Edamatsu, Nishida e Ouchi (1999). Nepomuceno, Oliveira e Lopes (2012) propõe como limites para $\mathrm{Gm}$ de 5,3 à 5,9 e para o Rm 1,14 à 1,30. Já, Edamatsu, Nishida e Ouchi (1999) apresentam como limites para o Gm de 3 à 7 e para Rm de 1 à 2.

Uma das características necessárias para a argamassa ser considerada autoadensável é a alta deformabilidade. A verificação desse comportamento ocorre por meio do ensaio de mini cone de espalhamento para argamassas onde são aferidas duas medições dos diâmetros pósespalhamento. Para a determinação do índice de deformabilidade $(\mathrm{Gm})$ é utilizado a equação 1.

$G_{m}=\left(\frac{D_{m}}{D_{0}}\right)^{2}-1$

Onde,

$\mathrm{G}_{\mathrm{m}}=$ índice de deformabilidade relativa,

$D_{m}=$ diâmetro médio do espalhamento,

$\mathrm{D}_{0}=$ diâmetro da parte inferior do cone.

A determinação do tempo de fluidez é realizada com a medição do tempo de escoamento de toda argamassa em um funil com dimensões pré-estabelecidas. $O$ índice de viscosidade relativa é obtido pela seguinte expressão.

$$
R_{m}=\frac{10}{\mathrm{t}}
$$

Onde,

$\mathrm{R}_{\mathrm{m}}$ = índice de deformabilidade relativa, $t=$ Tempo de escoamento no funil.

\section{RESULTADOS}

O estudo de composição dos agregados identificou como sendo a melhor proporção $40 \%$ de $C B C$ e $60 \%$ de areia média.A partir dessa proporção de agregado miúdo e $C B C$,foram elaboradas as argamassas autoadensáveis com CBC com relação 1:1 de aglomerante/agregado, em massa. Os resultados obtidos para as argamassas de relação 1:1 estão apresentados na Figura1. 
Figura 1. Comportamento da argamassa para os índices Gm e Rm.

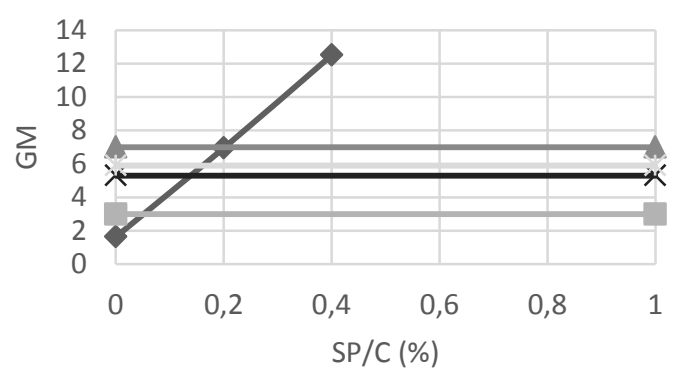

$\longrightarrow \mathrm{Gm}$

_L Lim. Inf. Edamatsu, Nishida e Ouchi (1999

_Lim. Sup. Edamatsu, Nishida e Ouchi (1999)

— Lim. Inf. Nepomuceno, Oliveira e Lopes (2012)

- Lim. Sup. Nepomuceno, Oliveira e Lopes (2012)

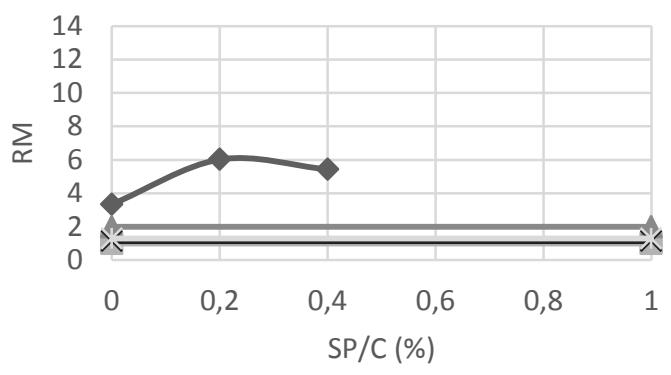

$\longrightarrow \mathrm{Rm}$

- Lim. Inf. Edamatsu, Nishida e Ouchi (1999)

LLim. Sup. Edamatsu, Nishida e Ouchi (1999)

— Lim. Inf. Nepomuceno, Oliveira e Lopes (2012)

- Lim. Sup. Nepomuceno, Oliveira e Lopes (2012)

Fonte: (Autor 2017)

Com os resultados obtidos pode-se identificar o traço com 0,2\% de aditivo superplastificante que se enquadrou nos limites de Edamatsu, Nishida e Ouchi (1999) para o índice $\mathrm{Gm}$, no entanto, este traço é rico em cimento não sendo recomendada a sua aplicação. Após, foram realizados os ensaios para as argamassas com relação 1:2, cujos resultados estão presentes na Figura 2. 
Figura 2. Comportamento das argamassas para os índices $\mathrm{Gm}$ e Rm na relação 1:2.

Fonte: (Autor 2017)
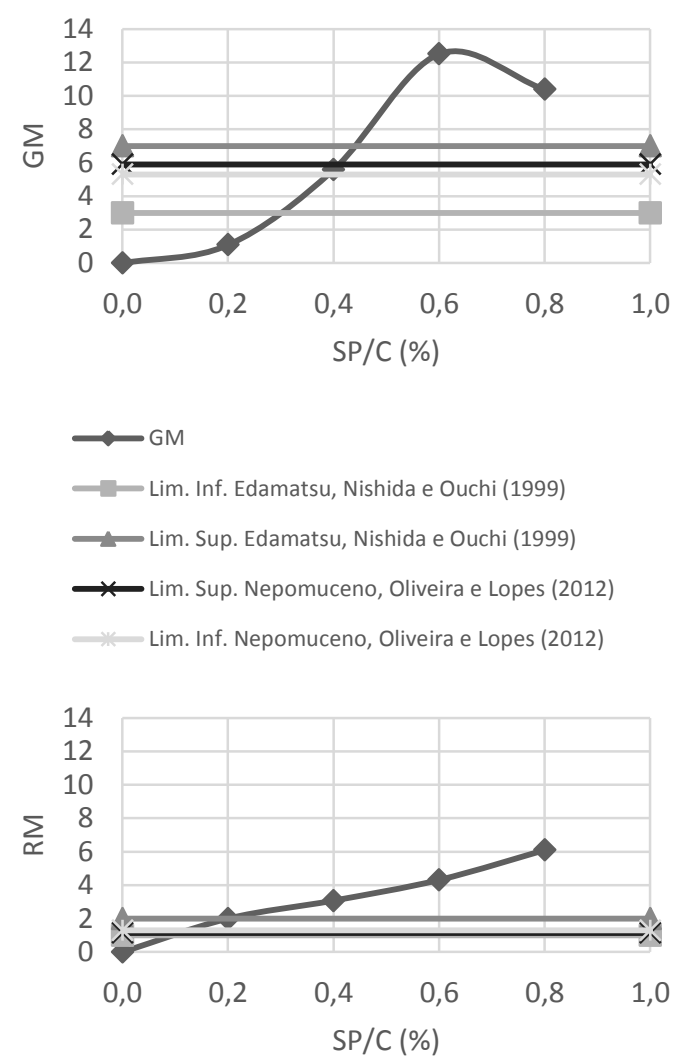

$\longrightarrow \mathrm{RM}$

L Lim. Inf. Edamatsu, Nishida e Ouchi (1999)

_ Lim. Sup. Edamatsu, Nishida e Ouchi (1999)

— Lim. Inf. Nepomuceno, Oliveira e Lopes (2012)

$\ldots$ Lim. Sup. Nepomuceno, Oliveira e Lopes (2012)

Ao analisar os gráficos presentes nas Figuras 1 e 2 foram identificadas duas argamassas com bons valores para os índices $\mathrm{Gm}$ e $\mathrm{Rm}$, sendo as com $0,2 \%$ e $0,4 \%$ de aditivo químico. $\mathrm{A}$ argamassa com $0,2 \%$ obteve um valor de 2 para o índice $\mathrm{Rm}$, obedecendo somente os limites propostos por Edamatsu, Nishida e Ouchi (1999). Já a argamassa com 0,4\% somente atendeu para o índice Gm os limites propostos por Edamatsu, Nishida e Ouchi (1999) e Nepomuceno, Oliveira e Lopes (2012), tendo um valor de 5,58.

Outro traço estudado foi com a relação 1:3. Neste caso, as argamassas feitas com essa relação não demonstraram a capacidade de se adensar devido ao grande volume de agregados, apresentando ausência total de fluidez. A relação adotada para as argamassas foi de 1:2, sendo essa a que obteve melhores resultados de fluidez e viscosidade.

Em seguida foram confeccionados traços comas dosagens de sílica ativa. As porcentagens de acréscimo da sílica foram de $5 \%, 7,5 \%$ e $10 \%$. Para o acréscimo de $5 \%$ de sílica ativa foram elaborados os gráficos presentes na Figura 3 para os índices $\mathrm{Gm}$ e Rm. 
Figura 3. Comportamento da argamassa com $5 \%$ de sílica ativa e CBC para os índices Gm e Rm.
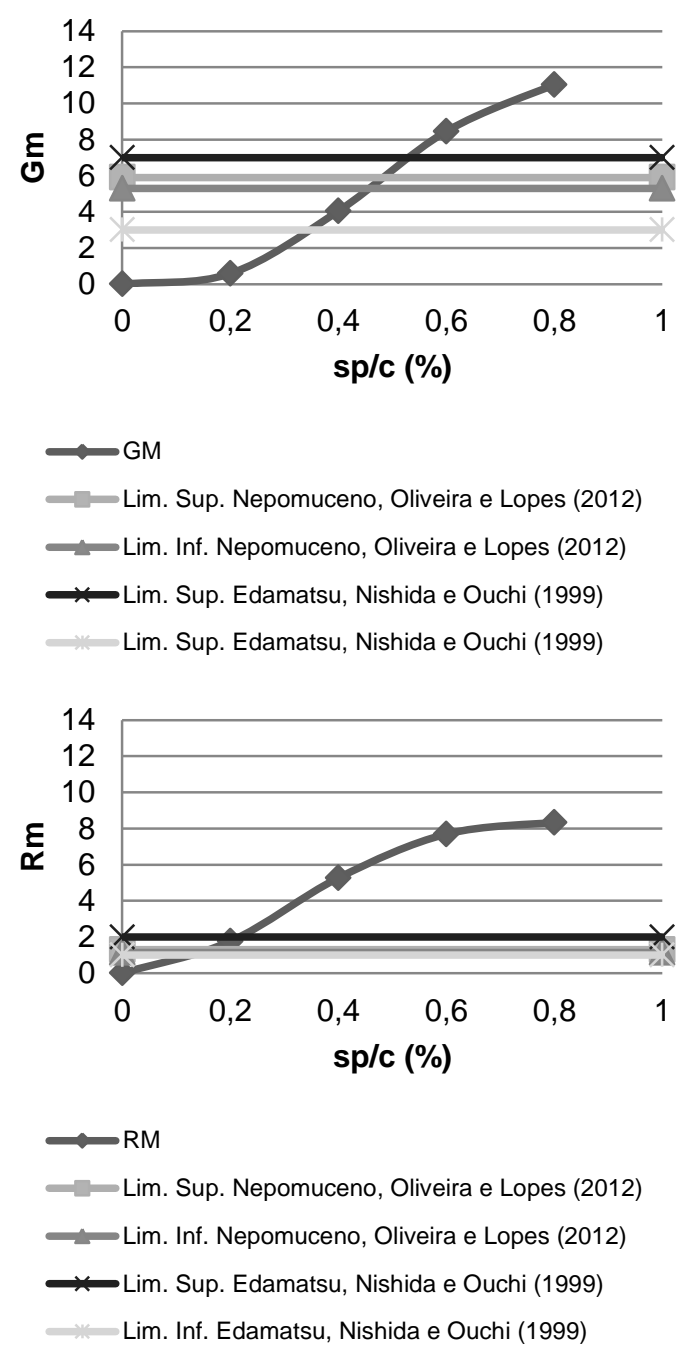

Fonte: (Autor 2017)

Com as informações dispostas nos gráficos da Figura 3, foi identificada a argamassa com $0,6 \%$ de aditivo químico atendendo os limites para o índice $\mathrm{Gm}$. Analisando visualmente as argamassas produzidas, foram eleitas as que possuem $0,4 \%$ e $0,6 \%$ de superplastificante em sua composição. As demais argamassas não apresentaram visualmente os requisitos necessários para serem consideradas autoadensáveis. Os resultados das argamassas com 7,5\% de sílica ativa são apresentados na Figura 4. 
Figura 4. Comportamento da argamassa com 7,5\% de sílica ativa e CBC para os índices $\mathrm{Gm}$ e Rm.
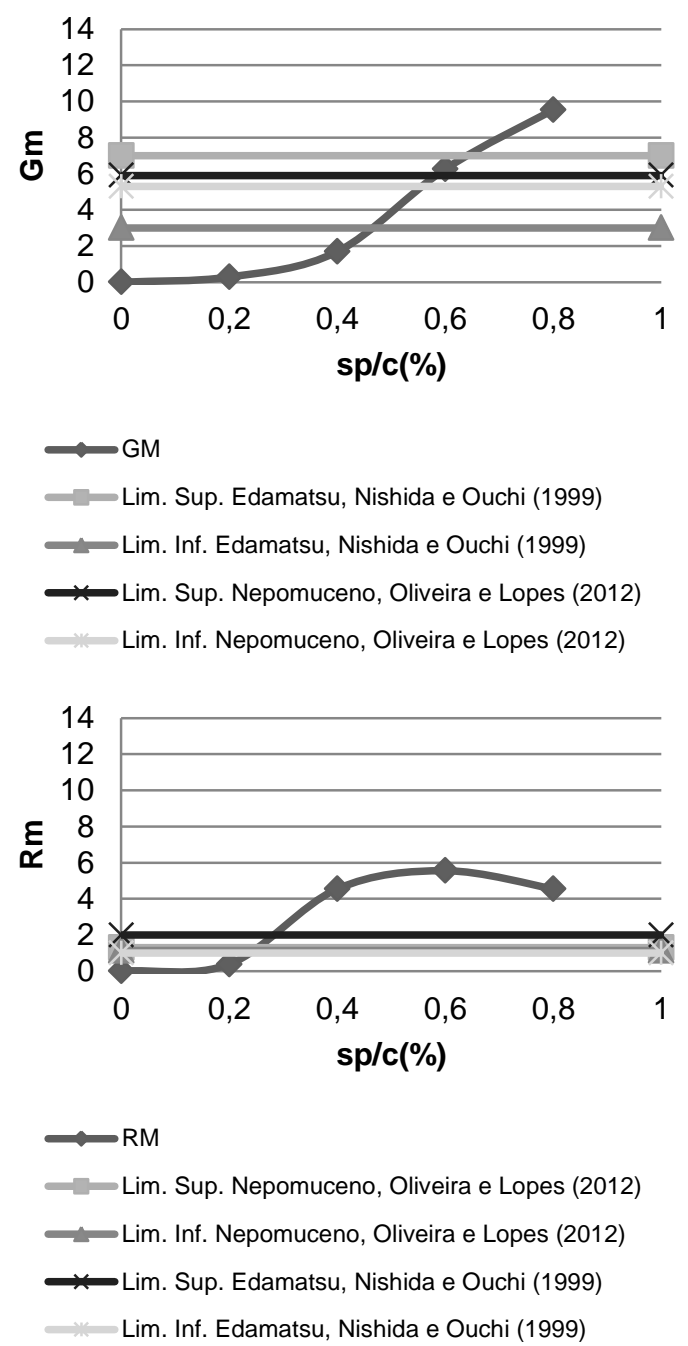

Fonte: (Autor 2017)

Por meio dos dados presentes na Figura 4, foi identificada a argamassa com $0,6 \%$ de superplastificante como a que atende os limites propostos por Edamatsu, Nishida e Ouchi (1999). Com a análise visual ocorreu a comprovação das características autoadensáveis dessa argamassa, a mesma não apresentou segregação e exsudação e foi capaz de fluir. As últimas argamassas elaboradas foram as com $10 \%$ de sílica ativa, a Figura 5 apresentam os resultados para os índices Gm e Rm. 
Figura 5. Comportamento da argamassa com $10 \%$ de sílica ativa e $C B C$ para os índices $G_{m}$ e $R_{m}$.
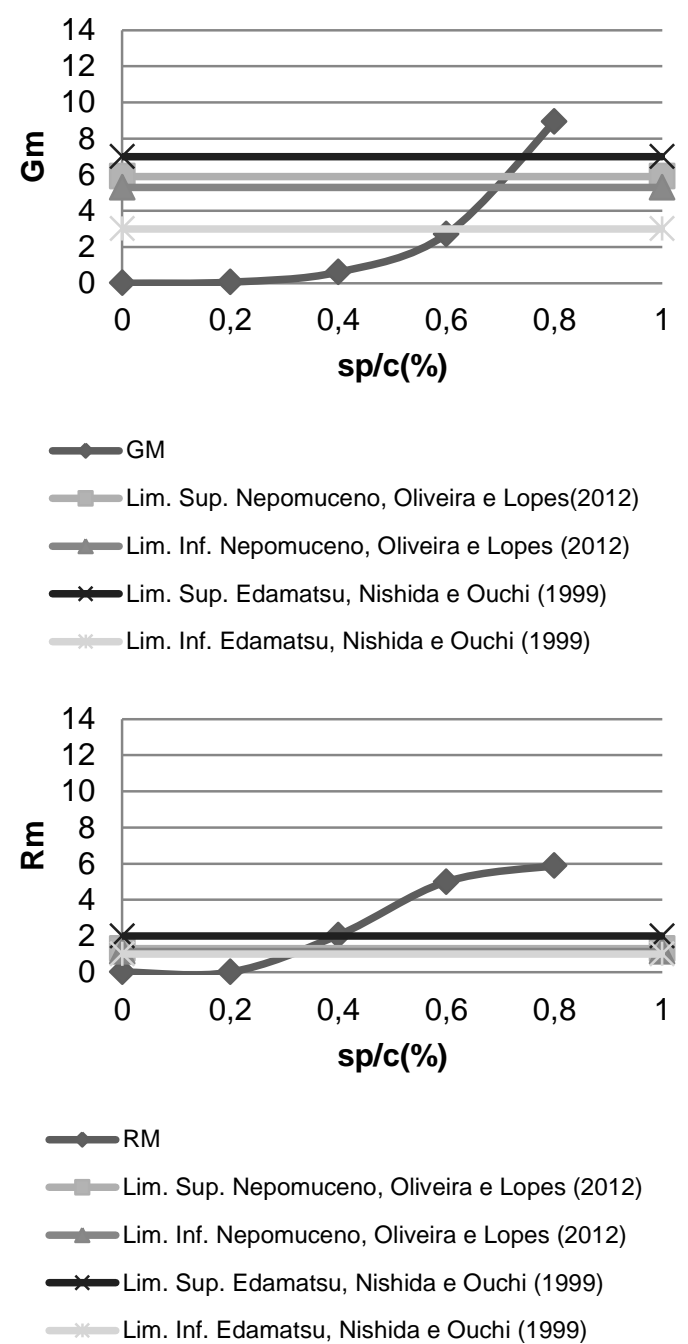

Fonte: (Autor 2017)

Com base nas informações apresentadas na Figura 5 foi possível identificar a argamassa com $0,6 \%$ de aditivo superplastificante como autoadensável, já que a mesma possui um índice $\mathrm{G}_{\mathrm{m}}$ dentro dos limites propostos por Edamatsu, Nishida e Ouchi (1999) e Nepomuceno, Oliveira e Lopes (2012). E com a realização da análise visual foi identificada a argamassa com $0,8 \%$ de superplastificante como sendo autoadensável, essa não apresentou segregação e exsudação e fluiu durante os ensaios.

Por meio dos resultados anteriormente demonstrados foram selecionadas as argamassas que atenderam os limites propostos para um dos índices e/ou que não foi identificada a presença da segregação e/ou exsudação visualmente.

As argamassas com CBC que foram selecionadas por meio da análise visual e dos limites: $0 \%$ de sílica ativa com $0,4 \%$ e $0,6 \%$ de aditivo químico; $5 \%$ de sílica com $0,4 \%$ e $0,6 \%$ de aditivo; $7,5 \%$ de sílica ativa com $0,4 \%$ e $0,6 \%$ superplastificante; e $10 \%$ de sílica ativa com $0,6 \%$ e $0,8 \%$ de aditivo. 


\section{DISCUSSÃO}

A argamassa autoadensável caracteriza-se por precisar de uma grande quantidade de finos para obter a capacidade de se adensar, logo, a CBC é um material que como foi comprovado pode ser aplicado nesse tipo de argamassa.

O uso da sílica ativa proporciona ganhos em viscosidade nas argamassas, no entanto, ocorreram dificuldades para conciliar os índices de $\mathrm{Gm}$ e $\mathrm{Rm}$ para os limites abordados. Outro ponto é que com o uso da sílica ativa nas argamassas com CBC ocorria uma diminuição no espalhamento das mesmas.

\section{CONSIDERAÇÕES FINAIS}

O melhor teor de substituição de areia por cinza do bagaço de cana-de-açúcar foi de 40\%, definido pelo menor índice de vazios obtido. A relação aglomerante/agregado que obtiveram melhores resultados para índice de deformabilidade $(\mathrm{Gm})$ e para índice de viscosidade relativa $(\mathrm{Rm})$ foi de 1:2.

Ao incorporar a sílica ativa nas argamassas ocorreu aumento da viscosidade e consequente redução da fluidez. Quanto maior a proporção de sílica ativa maior o tempo de escoamento no funil e menor a fluidez observada no ensaio de espalhamento. A argamassa que obteve os melhores resultados de viscosidade e fluidez foi a com $5 \%$ de adição de sílica ativa, sendo que a argamassa de referência (com cinza do bagaço de cana-de-açúcar e sem adição de sílica) não apresentou viscosidade e fluidez adequadas.

Dentre as argamassas com CBC elaboradas ao longo do estudos foram selecionadas por meio da análise visual e dos limites: $0 \%$ de sílica ativa com $0,4 \%$ e $0,6 \%$ de aditivo químico; $5 \%$ de sílica com $0,4 \%$ e $0,6 \%$ de aditivo; $7,5 \%$ de sílica ativa com $0,4 \%$ e $0,6 \%$ superplastificante; e $10 \%$ de sílica ativa com $0,6 \%$ e $0,8 \%$ de aditivo. Estas argamassas atenderam os limites e na análise visual não apresentaram exsudação e segregação e foram capazes de fluir.

\section{REFERÊNCIAS}

ASSOCIAÇÃO BRASILEIRA DE NORMAS TÉCNICAS. ABNT NBR NM 45. Agregados - Determinação da massa unitária e do volume de vazios. Rio de Janeiro, 2006.

EDAMATSU, H; NISHIDA, N; OUCHI, M. A rational mix-design method for self-compacting concrete considering interaction between coarse aggregate and mortar particles. In: FIRST INTERNATIONAL RILEM SYMPOSIUM ON SELF-COMPACTING CONCRETE, 1999, p. 309-320.

LIMA. C. I. VASCONCELOS. Concreto e suas inovações. Cadernos de graduação. Ciências exatas e tecnológicas. Faculdade Integrada Tiradentes. Maceió, Alagoas. 2014.

NEPOMUCENO, M; OLIVEIRA, L.; LOPES, S. Methodology for mix design of the mortar phase of selfcompacting concrete using different mineral additions in binary blends of powders. Construction and Building Materials. v. 26, n.1, p. 317-326, 2012, https://doi.org/10.1016/i.conbuildmat.2011.06.027.

OKAMURA, H.; OUCHI, M. Self-compacting concrete. Journal of Advanced Concrete Technology, Tokyo, vol. 1, n. 1, p. 5-15, abr. 2003, https://doi.org/10.3151/jact.1.5 